\title{
Special issue on Robotics: Science and Systems
}

\author{
Paul Newman · Nicolas Roy · Siddhartha Srinivasa
}

Published online: 9 August 2013

(C) Springer Science+Business Media New York 2013

Welcome to this special issue of Autonomous Robots. This volume is the second of two special journal issues compiled from the best papers presented at the eighth Robotics: Science and Systems (RSS) conference, held at the University of Sydney in July 2012. The other special issue was published by the International Journal of Robotics Research. Since its inception in 2005, the conference has continued to attract scientists working on the algorithmic and mathematical foundations of robotics, robotics applications, and analysis of robotic systems. We are excited to showcase five papers selected from RSS 2012. Although they are on diverse topics-assisted teleoperation, underwater terrain segmentation, multi-robot allocation, micro aerial vehicles, humanoid walking-they share a common theme of developing new theories and algorithms coupled with the commitment to demonstrate these insights on real robotic platforms, marrying the science and systems of robotics.

Hauser's work explores the problem of developing assistive user interfaces for controlling robotic manipulators. The paper combines estimating the user's intention via a predictor with motion replanning to be responsive to the user's needs.

P. Newman

Department Engineering Science, University of Oxford,

Parks Road, Oxford OX1 3PJ, UK

e-mail: pnewman@ robots.ox.ac.uk

N. Roy

Massachusetts Institute of Technology, 77 Massachusetts Avenue,

33-315, Cambridge, MA 02139, USA

e-mail: nickroy@mit.edu

S. Srinivasa $(\varangle)$

The Robotics Institute, Carnegie Mellon University,

5000 Forbes Avenue, Pittsburgh, PA 15213-3890, USA

e-mail: siddh@cs.cmu.edu
Douillard et al. study the segmentation of colorized point clouds of a seafloor acquired from an Autonomous Underwater Vehicle. Their key insight is to solve ground-object segmentation-distinguishing the slowly undulating seafloor from the clutter above-by analyzing the Fourier spectrum of the data.

Liu et al. explore the distributed multi-robot task-allocation problem, where a team of robots must negotiate the best assignment of tasks to robots. They present a provably efficient task-swapping mechanism and draw insightful parallels between approximate techniques developed in robotics and combinatorial optimization techniques.

Kushleyev et al. discuss the design and construction of smaller, better, and faster micro quadrotors. The latter half of the paper then describes algorithms for planning and control for groups of quadrotors in formation flight.

Sentis et al. describe the development of prioritized wholebody compliant controllers on their humanoid robot called Dreamer. The paper serves as a proof-of-concept of much theoretical and algorithmic work on compliant control, and describes the control architecture for rendering the theories on a real humanoid platform.

We hope you enjoy this special issue as much as we did pulling it together. We are grateful to the reviewers of both the conference and final journal versions of the papers, and to the editorial staff of Autonomous Robots for their patience and guidance. 